\title{
Detection of Glaucoma using ORB (Oriented FAST and Rotated BRIEF) Feature Extraction
}

\author{
Kazi Safayet Md. Shabbir ${ }^{1}$, Md. Imteaz Ahmed ${ }^{2}$, Marzan Alam $^{1}$ \\ ${ }^{1}$ Department of Mechatronics and Industrial Engineering, Chittagong University of Engineering and Technology, Chattagram- \\ 4349, Bangladesh \\ ${ }^{2}$ Department of Mechanical Engineering, Chittagong University of Engineering and Technology, Chattagram-4349, Bangladesh
}

Received: June 14, 2021, Revised: August 02, 2021, Accepted: August 09, 2021, Available Online: August 23, 2021

\begin{abstract}
This research was utilized to identify glaucoma, a type of eye illness. This endeavor necessitates the use of pictures from the fundus camera for image processing. This study reflects the effort done to detect glaucoma-affected eyes utilizing image feature extraction using Oriented FAST and Rotated BRIEF (ORB). ORB is a binary descriptor approach that is based on BRIEF and is highly fast. This technique is insensitive to picture noise and is invariant to any rotation. ORB is two orders of magnitude faster than SURF and performs similarly to SIFT. It is more efficient than other texture analysis methods. It is less computationally difficult than other approaches in the literature. This technique extracts features and detects texture by inspecting each pixel of the retina picture. It was trained on 160 fundus pictures of normal and glaucoma-affected retinas. After that, any healthy or glaucoma-affected eye may be easily recognized by obtaining an accurate eye picture. The results reveal that this technique has a precision and accuracy of more than $90 \%$.
\end{abstract}

Keywords: ORB, Glaucoma, Fundus, SIFT, Python.

This work is licensed under a Creative Commons Attribution-Non Commercial 4.0 International License.

\section{Introduction}

In 1622 English oculist Richard Bannister was the first person who found out the elevation of Intraocular Pressure (IOP) that causes tension in the eyeball and termed it as glaucoma [1]. Glaucoma causes blindness if it is ignored and there is no way of detecting an affected eye without taking a fundus image of the retina. As glaucoma does not have any symptoms and there is no single test to detect people with glaucoma [2] it became the third leading reason for blindness throughout the globe. A source implies that 4.5 million people across the world have been blinded because of glaucoma [3]. Based a surveillance study in 2004 in Dhaka, Bangladesh shows that approximately 586,000 people are definitely or probably suffering from glaucoma [4]. That's why early detection of glaucoma can be a great solution to prevent blindness. This leads to a screening-based program to classify glaucoma-affected retina. Screening-based programs require a retinal image of the patient and an optometrist will decide if the patient needs any medical attention furthermore. This is the only solution to detect glaucoma at its early stage and any kind of optical surgery (like laser treatment or implementing drainage) relating to glaucoma can be avoided to preserve the quality of the patient's life and medical cost as well. Present techniques to detect glaucoma are not cost-effective so that, implementation of these techniques can be expensive. If the methods are improved it can drastically reduce the cost and make the implementation easier.

The diagnosis of glaucoma can be done using three essential tests. Firstly, Tonometry includes the measurement of Intraocular Pressure (IOP). But the thing is there is no difference in the value of IOP so that it is not possible to discriminate between healthy and glaucomatous eyes [2]. Secondly, visual field testing is a subjective and most sensitive examination if used as a screening test [5]. But there is a handicap of this method, it needs comparatively more time to run the whole test, highly developed equipment, and an operator who will guide the subject during the test. Most of the patients found this task difficult to perform and $90 \%$ of the patients aging 40 years could perform the screening test properly. The success rate drastically decreases to $71 \%$ for patients aging more than 70 years [2]. Finally, the nerve layer or optic disc examination can be the procedure of choice for screening tests of glaucoma. To perform this test fundus camera or 3D imaging tool is necessary to detect the signs of glaucoma in the retina. 3D imaging tool has an advantage over the fundus camera as it can gather data of the optical disc on a 3D perspective measurement.

Cup depth of the retina is one of the most common indicators of glaucoma that can easily be measured. This is a more accurate approach to detect glaucoma. Besides being able to measure such things 3D imaging tools are expensive and not suitable for largescale implementation. Therefore, computer-aided diagnosis (CAD) using screening tests of fundus images is more acceptable because of its availability and cost-efficiency. In this paper, the fundus image has been used to detect glaucoma-affected eyes. The method used in this work is feature detection based on Oriented FAST and Rotating BRIEF (ORB) which is more suitable for this task than other feature extraction methods like SIFT or SURF [6]. This process uses each pixel of the image, unlike other methods that use specific regions of the image to perform the task.

The paper has been organized as follows, Section II includes a Literature review about the previous work done in this field. Section III describes the method that has been used to perform the task of detection. Section IV represents the result found in the experiment and the conclusion about it. 


\section{Literature Review}

All of the previous work that has been published can be categorized into two sections, fractionalizing the retinal objects, on the other hand, classifying it with the help of features in the image. To imply the first category segmentation is needed of the patient's retina so that it can get the structure of it. From the structure, some parameters define the healthy retina and glaucomatous retina through calculation. It is cupping that is damaged due to glaucoma in the retina. This is one of the main things that is considered as the main features alongside with image of the optic disc and blood vessels' retinal image.

In the early days, various Greek medical literature described glaucoma as an incurable disease [7]. But through numerous medical and technological researches now it has become curable. The first approach to cure this disease was to decrease IOP using drugs that were discovered in a study of intraocular hemorrhage control [8]. All the work was related to curing glaucoma but the hardest part was to detect it before it starts damaging the eye. In the late 19th century blue on yellow perimetry was a successful method of detecting glaucoma in its early stage [9].

Enlarging of the optical disc is what happens in the optical nerve system because of glaucoma. This is why the cup to disc ratio (CDR) varies for normal vs. affected eyes (See Fig. 1). Disc size does not affect detecting glaucoma and it was found in a study of open-angle glaucoma [10]. Moreover, emission of blood occurs in the optic disc and retinal disc, the retinal layer gets thinner, and the retinal nerve fiber layer (RNFL) gets defected. Calculating CDR and RNFL there is a method to detect glaucoma [11]-[12]. The ratio between the vertical diameter of the optic and disc is known as CDR, which is most commonly used for detecting glaucoma and must needed measurement parameter for segmentation.

CDR is very sensitive to glaucoma that has made an opportunity to examine it as this represents the deformation inside the optic cup. Detection of CDR can be automatically done that is proposed by a study [13]. The high value of CDR means a large optic disk, which happened due to glaucoma affection inside the retina [14]. But there are some exceptions found in some research like, CDR in some cases act like a continuous variable that is distributed as IOP [2]. This can turn into a conclusion like this, Small CDR valued patients might face vision loss or vice versa [15]. To avoid this kind of miscalculation CDR of both optic disc is needed to make sure if the disc size of the retina is large or not.
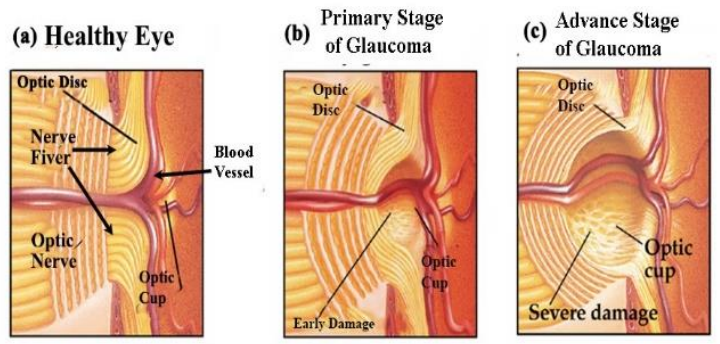

Fig. 1 Cupping in glaucomatous damage. a) Normal disc with small cup region, b) disc with early glaucoma, and c) disc with advanced glaucoma.

Accurate segmentation that has been taken from both of the eyes of the patient is mandatory for CDR-based methods used for detecting glaucoma [16]. Misdiagnosis may occur if there is an error in measuring CDR value or in segmentation, thus taking these values accurately is vital. It is very difficult to get the correct segmentation of the retinal image as the optic disc size varies according to optic cups. Perfect segmentation results in sensitive, specific, and accurate glaucoma detection and it is tough to achieve. A method was developed to estimate the amount of damage done by glaucoma, it used fundus images of the eye [17]. The difficulties arise because of the low contrast created by the blood vessels of the retina in the fundus image around the area of the optic cup/disc.

The specific field of this computer-aided diagnosis is a classification of the fundus image using image processing to achieve the highest accuracy possible. Several works had been done to achieve this goal. Using colored fundus Image automatic glaucoma detection method was proposed using Image processing [18]. Wavelet-based classification is used in a study to extract features of glaucoma for detection [19]. Another image processing-based work that has been done is Histogram of Oriented Gradients (HoG) using the Naive Bayes method of classification [20]. The automatic system for analyzing the retinal images to detect glaucoma was in 2014 [21]. This work was about the depth discontinuity in the retinal surface.

All the present works relating to glaucoma are based on the researches above. As the process of detecting glaucoma is convenient with image processing there have been several works done in this field. Various image processing method has been applied to analyze the texture of glaucoma or classify glaucoma from the fundus image. Texture analysis has been done using the Binary Robust Independent Elementary Feature (BRIEF) method [22]. BRIEF is a binary-based descriptor that has a high success rate in texture analysis. In this paper, the method used is a BRIEF-based feature detection and tracking system called ORB. The advantage of using this method is quick response, no issue with blood vessel contrast of the image, and higher accuracy than any methods that have been used till now to pursue this work. So, to get better accuracy and precision this method has been used in this study.

\section{Methodology}

The whole process is done using the python language and using library of it. The colored fundus image from the fundus camera is where the work starts from. The method using here uses pixel by pixel of the image that helps to gain more accuracy with less complexity. After splitting the image's colors using the green channel gave a spectacular result while gathering features of the images that has been used. Histogram equalization intensified the features of the images that are useful for extracting features.

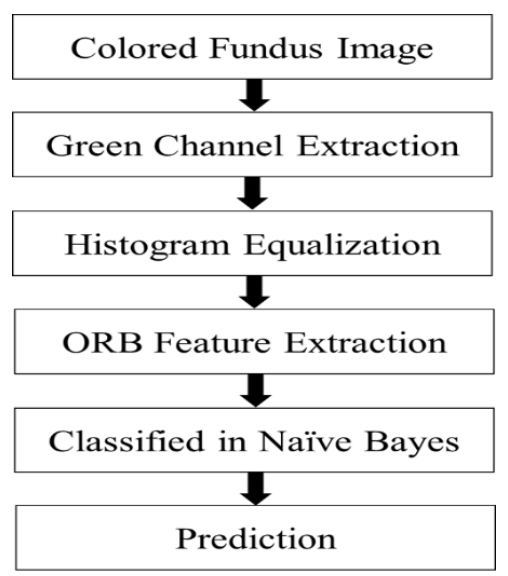

Fig. 2 Detecting Glaucoma using ORB 
After processing the image, Oriented FAST and rotated the BRIEF (ORB) method has used for extracting features. Then organized those extracted features in stacks and fed them to Naïve Bayes for classification and training the model. Finally, that model predicts images if they have glaucoma or they are healthy. The whole process is shown in Fig. 2.

\subsection{Image Preprocessing}

This section is divided into two steps. At first, green channel extraction and then equalization of the histogram. All these processes are done in a python language environment. Fig. 3 is an example image that will be used for further explanation. Applying all the steps used in this method into this image other steps can be easily described.

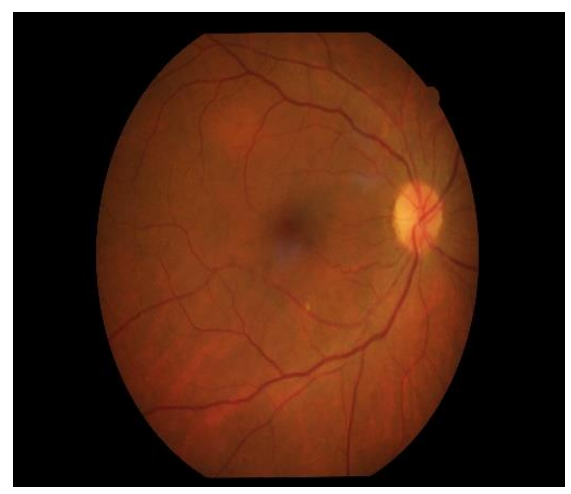

Fig. 3 Colored Fundus Image

\subsection{Green Channel Extraction}

First, it splits images using the library and then use various channels of colors Blue, Green, and Red. After that green channel gives better results in terms of extracting features than the other two channels. Using library read the colored fundus image at first, then using image slice a 2D array extracts green channel from the image. This is a part of pre-processing the image and getting it ready for feature extraction.

After using the green channel extraction operation of the fundus image it appears to be like Fig. 4.

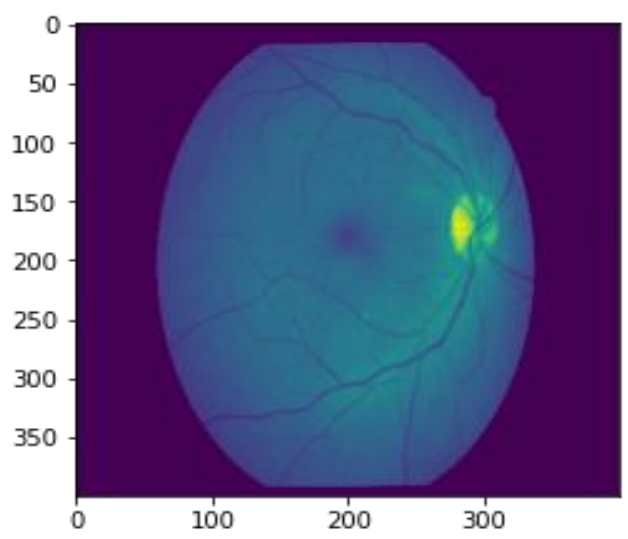

Fig. 4 Green Channel Extraction in Pre-processing the fundus image

This image was resized in $(400,400)$ pixels and then we use a split function from the library to get three separate channels. Then we used the green channel and that's why the image is greenish. But the image is not clear enough for extracting features from it, which is when the Histogram Equalization is required.

\subsection{Histogram Equalization}

Histogram equalization is required to make sure of using all the features an image contains that is important in image processing. It stretches the histogram of the image to get the right pixel value in all regions of the image so that it intensifies the clarity of the image so all the features are visible for extraction.

For histogram equalization, there are various functions. It has a dedicated function for histogram equalization but it has some limitations in a highly contrasted image where the image has a higher histogram region with both types of pixels: dark and bright. To solve this problem, Contrast Limited Adaptive Histogram Equalization (CLAHE) is used in our method. CLAHE requires a clip limit of the image and uses the title grid size of the photo to enhance its histogram. Using clip limit and grid size the image is distributed into small tiles. In OpenCV the default tiles size is $8 \times 8$ and this method uses this size as well. After getting those $8 \times 8$ pixel tiles it equalizes the histogram in that small region. To avoid any noise enhancement of the tiles contrast limiting is used that has a default value, 40. Before equalizing the histogram, it limits the contest value and distributes those clipped pixels evenly to further bins. If any artifacts appear in those $8 \times 8$ tiles border, using bilinear interpolation it is removed in this method of histogram equalization. Therefore, using this function in images with higher histograms results in a more evenly distributed histogram both in the foreground and the background of the image.

The green channel extracted image after applying CLAHE is shown in Fig. 5. It has become more visible and more detailed that is required for a better feature extraction process.

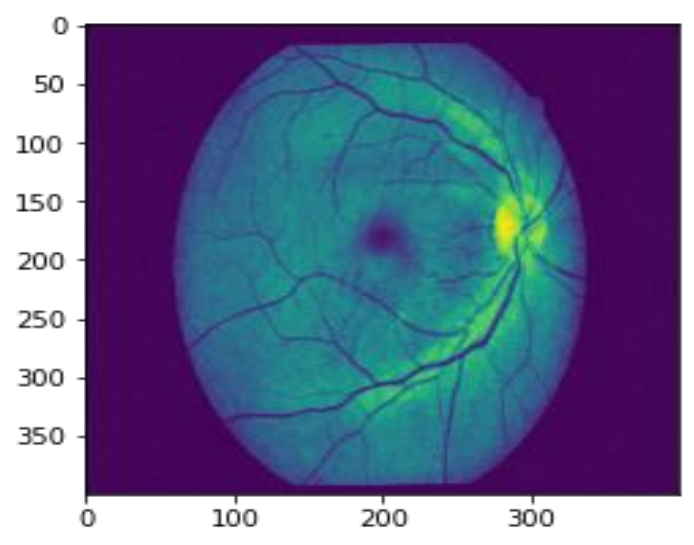

Fig. 5 Equalizing Histograms using CLAHE function

\subsection{Feature Extraction}

Oriented FAST and Rotated BRIEF (ORB) is our feature extraction method for texture analysis of the fundus image. ORB is a highly modified combination of two methods Features from Accelerated Segment Test (FAST) and Binary Robust Independent Elementary Feature (BRIEF). From FAST it gets key points as it is a corner detection method and from BRIEF, we get descriptor. ORB uses them to enhance their performance and overcome the limitations of FAST that is it can't calculate the orientation of the image.

Locating the patch of the corner in the center, it calculates the centroid based on intensity weight, taking vector from the corner point and centroid gives the direction. Therefore, orientation is achieved from it. With respect to $\mathrm{X}$ and $\mathrm{Y}$ moments of the rotation are calculated that have a radius of ' $\boldsymbol{r}$ ', which improves the invariance of rotation. Here ' $r$ ' represents the size 
of the path. Using the key points found in the Oriented FAST method BRIEF is steered to overcome its limitations of performance in terms of rotation. If the feature set of binary tests in BRIEF is ' $\mathrm{m}$ ' and the location is $\left(\boldsymbol{x}_{i}, \boldsymbol{y}_{i}\right)$ makes a $2 \times$ m matrix. The coordinates of the pixels are stored in ' $\mathrm{S}$ '. A rotation matrix $S_{\theta}$ is found using $\theta$ that implies the orientation of the patch.

In ORB it creates a progress table of previously calculated BRIEF patterns separating the angle with increments of 12 degrees $(2 \pi / 30)$. $\mathrm{S}_{\theta}$ will be used further as long as the key point orientation $\theta$ is used. When rotated according to those key points BRIEF gets more distributed and loses its important property of having higher variance and 0.5 mean value of each bit features. Plus point of having high variance is it helps the feature to be more discriminatory as it is response distinctively to inputs. There is another important property needed for the feature extraction which is having all the tests uncorrelated. To gain these absent properties ORB method performs a greedy search among all the binaries found after rotating the BRIEF according to key points. This greedy search finds out all the possible binaries that have large variance and means closer to 0.5 and makes these tests uncorrelated. This modified BRIEF is known as rBRIEF or Rotated BRIEF. To achieve matching in the descriptor, multi-probe Locality Sensitive Hashing (LSH) is used that has improved output than usual LSH.

To use ORB in python it just needed to call as function. This function extracts features from the pre-processed image. The number of features extracted in this method is 500 . Key points and descriptors are found as output after computing them from the pre-processed fundus image, this method used the descriptor for further use. The number of features taken in descriptors here is [0:200]. Then those descriptors are stacked from each image one after another after running it through the ORB function. With 160 images going through the ORB function the number of features in stacked descriptor is 6400. Fig. 6 shows how the image looks after running it through the ORB feature extractor function.

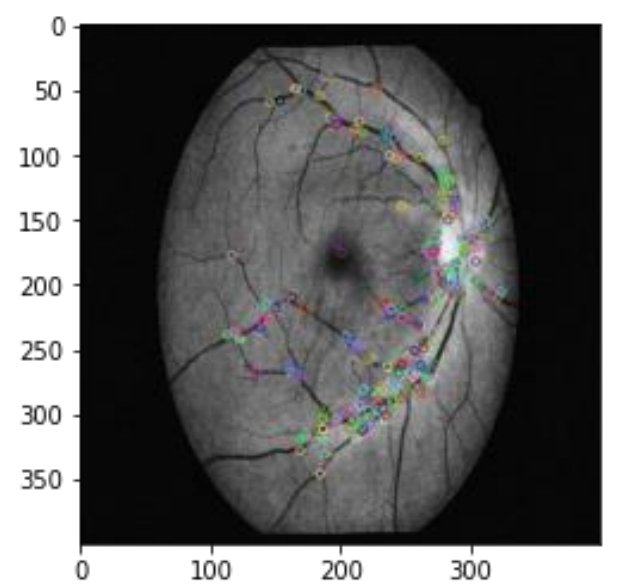

Fig. 6 Feature extraction using ORB method

After stacking those descriptors, a model is trained using machine learning. Here 80 fundus images were healthy and the other 80 were glaucoma-affected images. Classifying those images further work has been done.

\subsection{Classification}

Gaussian Naïve Bayes is a popular method of classification that can be easily used in Python through 'sklearn' library's datasets. Naïve Bayes is a technique used in statistical classification. It is based on Bayes Theorem which is used for predicting an outcome based on the data given. This simplifies the Bayes theorem and uses that for prediction and this is why it is known as Naïve Bayes. The equation is as follow:

$$
f\left(\frac{h}{D}\right)=\frac{f\left(\frac{D}{h}\right) f(h)}{f(D)}
$$

Here, $f(D)$ represents the probability of the data D regardless of the hypothesis that is prior probability here.

$f(h)$ Represents the probability of $h$ being true, which means hypothesis in regardless of given data. This is the prior probability of $h$.

$f\left(\frac{D}{h}\right)$ is the probability of $h$ being true on basis of the data $\mathrm{D}$ given. It is called posterior probability.

$f\left(\frac{h}{D}\right)$ is the probability of $h$ being true based on given the data $\mathrm{D}$. this is also known as posterior probability.

First of all, it calculates the prior probabilities for the class given. Secondly, it finds any similar probability for that label of the given class. Thirdly, it puts all those values in the Bayes formula to find out posterior probability. Lastly, it finds out which class shows the higher possibility of being true, then marks it as result.

In this work, we fed data as features that are found from ORB extraction and trained our model using Naïve Bayes. The desired output for detecting glaucoma is defined as Glaucoma $=0$, Healthy $=1$. Therefore, the trained model will show the result as 0 or 1 after processing the image used as input and predict if that image contains glaucoma or not.

\section{Simulation Results \& Discussion}

The method proposed above has been applied with the help of the fundus image collected. This helped to train the model properly in a python-based environment 'Jupyter Notebook'. To train this model we used 80 healthy fundus images and 80 glaucoma-affected fundus images. With the help of the ORB feature extraction method, we took [0:200] features and kept them in a descriptor. Then stacking those one by one in an array helped to train the model with the help of machine learning. Then the model used Gaussian Naïve Bays to predict normal or healthy eye and glaucoma affected eye based on the fundus image used as input.

To validate this classifier we calculated error rate, specificity, precision, and recall/sensitivity value. To calculate these values, 30 glaucoma-affected fundus images and 30 healthy fundus images were inputted into the trained model for prediction. This is what was found shown in Table 1.

Table 1 Confusion matrix of gaussian naïve bayes using ORB extraction method

\begin{tabular}{|c|c|c|c|}
\hline & $\begin{array}{c}\text { Predicted } \\
\text { Glaucoma }\end{array}$ & $\begin{array}{c}\text { Predicted } \\
\text { Healthy }\end{array}$ & $\begin{array}{c}\text { Total } \\
\text { Act. }\end{array}$ \\
\hline Actually Glaucoma & $\mathrm{TP}=28$ & $\mathrm{FP}=2$ & 30 \\
\hline Actually Healthy & $\mathrm{FN}=3$ & $\mathrm{TN}=27$ & 30 \\
\hline Total & & & 60 \\
\hline
\end{tabular}

In ORB key points and descriptors are found, descriptors are what stores features from the images using rBRIEF method. These descriptors are used to train the model and these are the things that help to predict the input image. In Table 1, TP 
represents the true positive numbers \& FP means the false positive numbers. Which means running 30 previously known glaucoma affected images as input in the prediction model we get 28(TP) correct result finding them as glaucoma affected and 2(FP) incorrect finding them as healthy. Similarly, for 30 healthy images running into the model as input, false-negative was 3 images, which means 3 images are detected as glaucoma affected though they are healthy, and 27 images are found true negative, which means correctly predicted them as healthy image.

Here,

$$
\begin{aligned}
& \text { Accuracy }=\frac{T P+T N}{T o t a l} \ldots \ldots \ldots \ldots \ldots . \ldots(2)=91.67 \% \\
& \text { Error rate }=\frac{F P+F N}{T o t a l} \ldots \ldots \ldots \ldots \ldots . \ldots(3)=8.3 \% \\
& \text { Specificity }=\frac{T N}{\text { Total Act. Healthy } \ldots . .(4)=90 \%} \\
& \text { Precision }=\frac{T P}{\text { Total Act. Glaucoma } \ldots \ldots . .(5)=93.3 \%} \\
& \text { Recall }=\frac{T P}{T P+F N} \ldots \ldots \ldots \ldots \ldots \ldots . . \ldots(6)=90.32 \%
\end{aligned}
$$

Table 2 Analyzing the performance of different methods

\begin{tabular}{|c|c|c|c|c|}
\hline Methods & Recall & Specificity & Accuracy & Precision \\
\hline Wavelet & 85.45 & 87.54 & 86.67 & 83.33 \\
\hline WBCT & 81.34 & 88.73 & 84.53 & 85.54 \\
\hline Contourlet & 74.36 & 80.20 & 78.84 & 76.84 \\
\hline HoG & 88.89 & 75 & 75 & 89.20 \\
\hline ORB & 90.32 & 90 & 91.67 & 93.3 \\
\hline
\end{tabular}

Table 3 Comparing ORB with other studies

\begin{tabular}{|c|c|c|c|c|c|}
\hline Author & Methodology & $\begin{array}{c}\text { Total } \\
\text { Image }\end{array}$ & $\begin{array}{c}\text { Classification } \\
\text { method }\end{array}$ & Accuracy & Recall \\
\hline $\begin{array}{c}\text { Vock et al. } \\
\text { pixel } \\
\text { intensity, } \\
\text { spectran and } \\
\text { texture } \\
\text { features }\end{array}$ & 200 & $\begin{array}{c}\text { NB, SVM, } \\
\text { KNN }\end{array}$ & $86 \%$ & N/A \\
\hline $\begin{array}{c}\text { Dua et al. } \\
{[19]}\end{array}$ & $\begin{array}{c}\text { Features of } \\
\text { wavelet } \\
\text { energy }\end{array}$ & 60 & $\begin{array}{c}\text { NB, SVM, } \\
\text { Sandom } \\
\text { forest }\end{array}$ & $86.67 \%$ & $85.54 \%$ \\
\hline $\begin{array}{c}\text { Krishnan } \\
\text { et al. [23] }\end{array}$ & $\begin{array}{c}\text { DWT and } \\
\text { Higher order } \\
\text { spectra trace } \\
\text { transform }\end{array}$ & 60 & SVM & $91.70 \%$ & $90 \%$ \\
\hline $\begin{array}{c}\text { Kolar } \\
\text { et al. [24] }\end{array}$ & $\begin{array}{c}\text { Fractal } \\
\text { features }\end{array}$ & 30 & SVM & $74 \%$ & N/A \\
\hline $\begin{array}{c}\text { Townsend } \\
\text { et al. [25] }\end{array}$ & $\begin{array}{c}\text { Detection } \\
\text { based on } \\
\text { HTR }\end{array}$ & 200 & SVM & $87.50 \%$ & N/A \\
\hline $\begin{array}{c}\text { Nirmal } \\
\text { et al. [20] }\end{array}$ & $\begin{array}{c}\text { Features of } \\
\text { HoG based } \\
\text { detection }\end{array}$ & 101 & NB & $75 \%$ & $88.89 \%$ \\
\hline $\begin{array}{c}\text { Proposed } \\
\text { method }\end{array}$ & $\begin{array}{c}\text { ORB feature } \\
\text { extraction } \\
\text { method }\end{array}$ & 160 & $\begin{array}{c}\text { GNB } \\
\text { (Python) }\end{array}$ & $91.67 \%$ & $90.32 \%$ \\
\hline
\end{tabular}

Comparing these values with other works previously done we get that accuracy of our work is better than them. The accuracy of Wavelet-based classification is $86.67 \%$, Contourletbased work had $78.84 \%$, WBCT had $84.53 \%$, HoG feature had $75 \%$ based on their confusion matrix provided. This ORB method Gained $91.6 \%$ accuracy. Recall of Wavelet-based classification is $85.45 \%$, Contourlet-based work had $74.36 \%$, WBCT had $81.34 \%$, HoG feature had $88.89 \%$ based on their confusion matrix provided. This ORB method Gained $93.32 \%$ recall. Specificity of Wavelet-based classification is $87.54 \%$, Contourlet-based work had $80.20 \%$, WBCT had $88.73 \%$, HoG feature had $75 \%$ based on their confusion matrix provided. This ORB method gained a $91.6 \%$ recall rate. Calculating the error rate of $\mathrm{HoG}$ based classification of its confusion matrix the rate found is $10 \%$ where ORB has an $8.3 \%$ error rate.

\section{Conclusion}

From this paper, it is clear that ORB feature extraction has higher accuracy, less complexity, highest sensitivity, and lowest error rate. The reason behind using this method is it's faster than other methods used before and less complex to use. The accuracy in texture analysis is high as well. Moreover, SURF and SIFT methods are patented, so paying the patent holders is required to use those methods. Because of using Gaussian Naïve Bayes in python, the prediction method becomes easier. OpenCV has various mathematical functions that are easier to use in python comparing to other platforms. ORB overcame all the limitations of the FAST and BRIEF method and faster than other feature extraction. The accuracy gained by the model we trained from 160 images is $91.67 \%$ and sensitivity or recall is $90.32 \%$ that is higher than other compared studies. Moreover, ORB can be used in any low-powered device, these reasons are what makes it a better choice than other methods.

\section{References}

[1] Banister, R., 1971. A treatise of one hundred and thirteene diseases of the eyes (No. 297). Theatrum Orbis Terrarum.

[2] Rotchford, A., 2005. What is practical in glaucoma management?. Eye, 19(10), pp.1125-1132.

[3] www.sightsavers.org/protecting-sight/what-is glaucoma/ Accessed on 24.04.2021

[4] Rahman, M.M., Rahman, N., Foster, P.J., Haque, Z., Zaman, A.U., Dineen, B. and Johnson, G.J., 2004. The prevalence of glaucoma in Bangladesh: a population based survey in Dhaka division. British Journal of Ophthalmology, 88(12), pp.1493-1497.

[5] Katz, J., Sommer, A., Gaasterland, D.E. and Anderson, D.R., 1991. Comparison of analytic algorithms for detecting glaucomatous visual field loss. Archives of Ophthalmology, 109(12), pp.1684-1689.

[6] Rublee, E., Rabaud, V., Konolige, K. and Bradski, G., 2011, November. ORB: An efficient alternative to SIFT or SURF. In 2011 International Conference on Computer Vision (pp. 2564-2571). IEEE.

[7] Laios, K., Moschos, M.M. and Androutsos, G., 2016. Glaucoma and the origins of its name. Journal of Glaucoma, 25(5), pp.e507-e508.

[8] Krupen, T., 1997. Autonomic drugs: controlling the inflow. van Buskirk EM, Shields MB, eds, 100, pp.262-71.

[9] Johnson, C.A., Adams, A.J., Casson, E.J. and Brandt, J.D., 1993. Blue-on-yellow perimetry can predict the development of glaucomatous visual field loss. Archives of Ophthalmology, 111(5), pp.645-650. 
[10] Jonas, J.B., Gusek, G.C. and Naumann, G.O., 1988. Optic disc morphometry in chronic primary open-angle glaucoma. Graefe's Srchive for Clinical and Experimental Ophthalmology, 226(6), pp.522-530.

[11] Nayak, J., Acharya, R., Bhat, P.S., Shetty, N. and Lim, T.C., 2009. Automated diagnosis of glaucoma using digital fundus images. Journal of Medical Systems, 33(5), pp.337-346.

[12] Muramatsu, C., Fujita, H., Nakagawa, T., Sawada, A., Yamamoto, T. and Hatanaka, Y., 2011. Automated determination of cup-to-disc ratio for classification of glaucomatous and normal eyes on stereo retinal fundus images. Journal of Biomedical Optics, 16(9), p.096009.

[13] Wolfs, R.C., Ramrattan, R.S., Hofman, A. and de Jong, P.T., 1999. Cup-to-disc ratio: ophthalmoscopy versus automated measurement in a general population: The Rotterdam Study. Ophthalmology, 106(8), pp.1597-1601.

[14] Varma, R., Steinmann, W.C. and Scott, I.U., 1992. Expert agreement in evaluating the optic disc for glaucoma. Ophthalmology, 99(2), pp.215-221.

[15] Douglas, G.R., GR, D. and SM, D., 1974. A correlation of fields and discs in open angle glaucoma. Can $J$ Ophthalmol, 9(4):391-8

[16] Lim, C.S., O'brien, C. and Bolton, N.M., 1996. A simple clinical method to measure the optic disc size in glaucoma. Journal of Glaucoma, 5(4), pp.241-245.

[17] Spaeth, G.L., Henderer, J., Liu, C., Kesen, M., Altangerel, U., Bayer, A., Katz, L.J., Myers, J., Rhee, D. and Steinmann, W., 2002. The disc damage likelihood scale: reproducibility of a new method of estimating the amount of optic nerve damage caused by glaucoma. Transactions of the American Ophthalmological Society, 100, p.181.
[18] Bock, R., Meier, J., Nyúl, L.G., Hornegger, J. and Michelson, G., 2010. Glaucoma risk index: automated glaucoma detection from color fundus images. Medical Image Analysis, 14(3), pp.471-481.

[19] Dua, S., Acharya, U.R., Chowriappa, P. and Sree, S.V., 2011. Wavelet-based energy features for glaucomatous image classification. IEEE Transactions on Information Technology in Biomedicine, 16(1), pp.80-87.

[20] Nirmala, K., Venkateswaran, N. and Kumar, C.V., 2017, November. HoG based Naive Bayes classifier for glaucoma detection. In TENCON 2017-2017 IEEE Region 10 Conference (pp. 2331-2336). IEEE.

[21] Joshi, G.D., 2014. Automatic retinal image analysis for the detection of glaucoma. International Institute of Information Technology.

[22] Mohammad, S. and Morris, D.T., 2015, May. Texture analysis for glaucoma classification. In 2015 International Conference on BioSignal Analysis, Processing and Systems (ICBAPS) (pp. 98-103). IEEE.

[23] Krishnan, M.M.R. and Faust, O., 2013. Automated glaucoma detection using hybrid feature extraction in retinal fundus images. Journal of Mechanics in Medicine and Biology, 13(01), p.1350011.

[24] Kolář, R. and Jan, J., 2008. Detection of glaucomatous eye via color fundus images using fractal dimensions. Radioengineering, 17(3), pp.109-114.

[25] Townsend, K.A., Wollstein, G., Danks, D., Sung, K.R., Ishikawa, H., Kagemann, L., Gabriele, M.L. and Schuman, J.S., 2008. Heidelberg Retina Tomograph 3 machine learning classifiers for glaucoma detection. British Journal of Ophthalmology, 92(6), pp.814-818. 